\title{
Microwave assisted synthesis, antifungal activity, DFT and SAR study of 1,2,4-triazolo[4,3-a]pyridine derivatives containing hydrazone moieties
}

\author{
Jin-Xia Mu ${ }^{1 *}{ }^{*}$, Yan-Xia Shi ${ }^{3+}$, Hong-Ke Wu ${ }^{2}$, Zhao-Hui Sun ${ }^{2}$, Ming-Yan Yang ${ }^{2}$, Xing-Hai Liu ${ }^{2^{*}}$ and Bao-Ju Li ${ }^{3^{*}}$
}

\begin{abstract}
Background: The increasing prevalence of multi-drug resistant fungal infections has encouraged the search for new antifungal agents. Hydrazone derivatives always exhibited diversity activities, including antifungal, anti-inflammatory, anti-oxidation, anti-cancer activity. Regarding the heterocyclic moiety, 1,2,4-triazolo[4,3-a]pyridine derivatives also display broad activities, such as antifungal activity, anticonvulsant activity, herbicidal activity, antimicrobial activity and anticancer activity.

Results: A series of novel 1,2,4-triazolo[4,3-a]pyridine derivatives containing hydrazone moiety were designed and synthesized from 2,3-dichloropyridine, hydrazine hydrate by multi-step reactions under microwave irradiation condition, and their structures were characterized by FT IR, ${ }^{1} \mathrm{H} \mathrm{NMR},{ }^{13} \mathrm{C} \mathrm{NMR},{ }^{19} \mathrm{~F} \mathrm{NMR}, \mathrm{MS}$ and elemental analysis. The antifungal activities of title compounds were determined. The results indicated that some of the title compounds exhibited good antifungal activity. Furthermore, DFT calculation was carried out for studying the structure-activity relationship (SAR).
\end{abstract}

Conclusion: A practical synthetic route to obtain 1,2,4-triazolo[4,3-a]pyridine derivatives is presented. This study suggests that the 1,2,4-triazolo[4,3-a]pyridine derivatives exhibited good antifungal activity.

Keywords: 1,2,4-Triazolo[4,3-a]pyridine, Hydrazone, Microwave assisted synthesis, Antifungal activity, DFT

\section{Background}

Nowadays, the synthesis of nitrogen containing heterocycles is an important direction in the fields of pesticidal chemistry [1-3], medicinal chemistry [4], polymer chemistry [5], coordination chemistry [6] and industrial chemistry [7]. 1,2,4-Triazole derivatives and pyridine derivatives often display broad and diverse biological activities [810]. Some reports found that fused heterocycles generally

\footnotetext{
*Correspondence: mujinxiajl@sina.com; xhliu@zjut.edu.cn; libaoju@caas.cn

†Jin-Xia Mu and Yan-Xia Shi contributed equally to this work ${ }^{1}$ Department of Environmental Engineering, China Jiliang University, Hangzhou 310018, Zhejiang, China

${ }^{2}$ College of Chemical Engineering, Zhejiang University of Technology, Hangzhou 310014, China

${ }^{3}$ Institute of Vegetables and Flowers, Chinese Academy of Agricultural Sciences, Beijing 100014, China
}

displayed mixed properties of the corresponding single heterocycles. Many references proved that the fusing of triazole and pyridine rings was a good way to produce highly active compounds, such as herbicidal [11, 12], antifungal [13, 14], anticonvulsant [15], antibacterial activity [16]. Furthermore, the acylhydrazone structure is considered an important pharmacophore in drug discovery [17]. In the past years, there have been many reports in the literature for the synthesis and biological activities of hydrazone derivatives [18-20], such as acaricidal, anti-cancer, insecticidal, antifungal, antibacterial, antimicrobial and antileishmanial activity. In addition, hydrazones also are very useful starting materials in bioactive heterocycles, such as $\beta$-lactams, pyrazoles, and pyrazines.

In our previous work, many 1,2,4-triazolo[4,3-a]pyridine derivatives were designed and synthesized, which 
exhibit excellent and diverse activity [11-14]. In line with our continuous efforts to synthesize bioactive lead compounds for crop protection [21-27], the title 1,2,4-triazolo[4,3-a]pyridine derivatives were designed and synthesized by introducing acylhydrazone pharmacophore into the lead compound (Scheme 1).

\section{Results and discussion}

\section{Synthesis}

The key intermediate 8 -chloro- $[1,2,4]$ triazolo[4,3-a]pyridine-3-carbohydrazide was synthesized according to the Ref. [28]. Microwave technology was applied to the synthetic reaction to shorten the reaction time and increase the yield. First, the one pot synthesis of intermediate 1 under microwave irradiation was applied, but the result was not better than that of conventional condition. Then intermediate $\mathbf{1}$ was cyclized with diethyl oxalate lead to the intermediate 2 by a condensation reaction. At last, the 8-chloro-[1, 2, 4] triazolo[4,3-a]pyridine-3-carbohydrazide reacted with different aldehyde in ethanol was synthesized under microwave irradiation conditions. This reaction was completed with higher yields compared with the conventional mode of heating. The synthetic route is showed in Scheme 2.
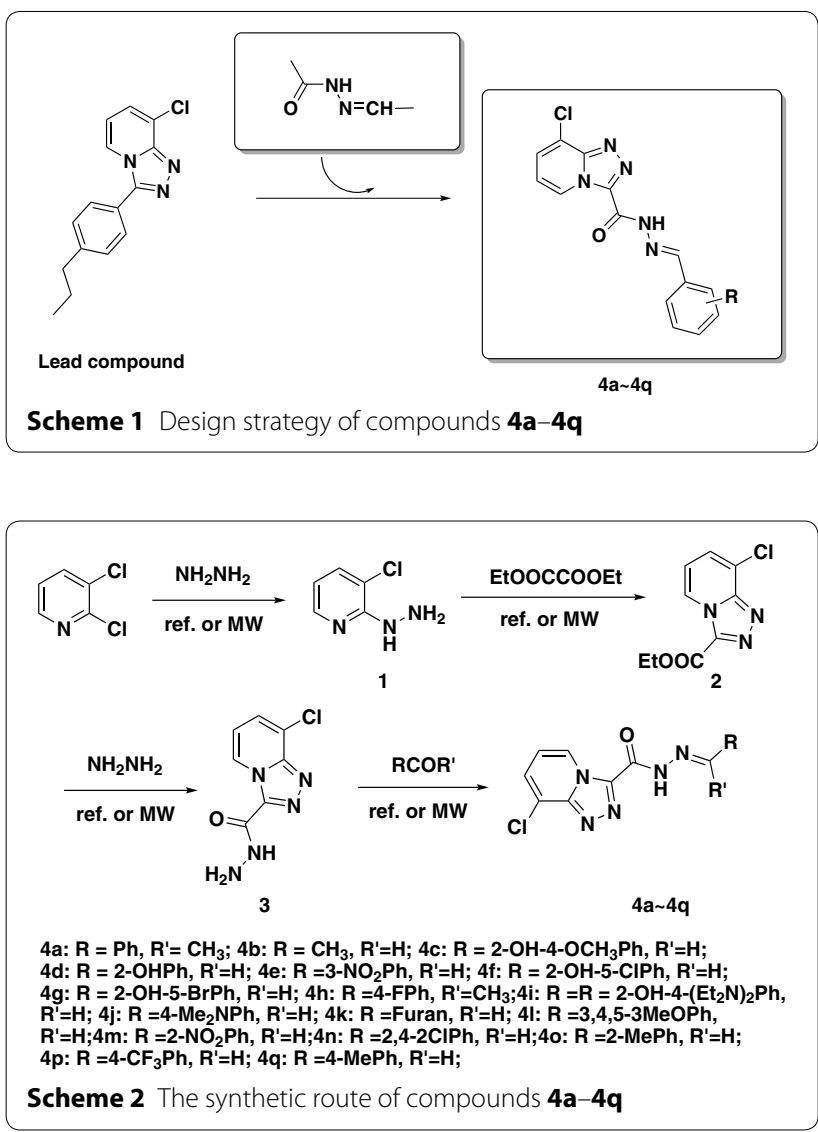

The reaction parameters were optimized for the synthesis of title compounds. The title compounds were prepared from 8-chloro-[1, 2, 4] triazolo[4,3-a]pyridine-3-carbohydrazide and substituted aldehyde under microwave irradiation condition, leading to the desired compounds in $82-94 \%$ yields. The compound 4d was chosen as a model reaction under different conditions. Several key reaction conditions were studied, such as reaction temperature, reaction times, reaction mode (conventional or microwave irradiation). The results are illustrated in Table 1. From Table 1, it is indicated that the microwave irradiation method allowed a shorter reaction time, compared with the room temperature or reflux condition. Also we can see that the yield of compound 4d is higher under microwave irradiation condition than that of room temperature or reflux condition. Under the microwave irradiation condition, the yield increase, when the reaction time is prolonged (10 $\mathrm{min})$. Meanwhile, the longer reaction time held higher yield. From the Table 1, the best reaction condition is $78^{\circ} \mathrm{C}$ and $10 \mathrm{~min}$.

All the compounds were identified and characterized by FTIR, ${ }^{1} \mathrm{H}$ NMR, ${ }^{13} \mathrm{C}$ NMR, ${ }^{19} \mathrm{~F}$ NMR, MS and elemental analysis. In the ${ }^{1} \mathrm{H}$ NMR spectra of target compounds, all the $-\mathrm{NH}$ proton signals of the title compounds can be found around 10-13 ppm. The appearance of signals at $\sim 7.0, \sim 7.5$ and $\sim 9.3 \mathrm{ppm}$ are assigned to pyridine ring. The infrared spectrum of acyl hydrozone derivatives 4 showed absorption bands at $3139-3500 \mathrm{~cm}^{-1}$ for $\mathrm{N}-\mathrm{H}$ stretching. The characteristic stretching vibrations $v(C=O)$ and $v(\mathrm{C}=\mathrm{N})$ appears at $1657-1724,1618-1686 \mathrm{~cm}^{-1}$ respectively. Meanwhile, most of the title compounds exhibited the $\mathrm{M}+\mathrm{H}^{+}$peak in the ESI-MS results.

\section{Antifungal activities and SAR}

The antifungal activities of compound $\mathbf{4 a - 4 q}$ were evaluated in vivo at $100 \mu \mathrm{g} / \mathrm{mL}$ against Stemphylium lycopersici (Enjoji) Yamamoto (SL), Fusarium oxysporum sp. Cucumebrium (FO) and Botrytis cinerea (BC) and the bioassay results were listed in Table 2. From Table 2,

Table 1 Comparison of yields of $4 \mathrm{~d}$ through methods with or without microwave irradiation

\begin{tabular}{llllll}
\hline Entry & Solvent & Method & Time $(\mathbf{m i n})$ & Temperature $/{ }^{\circ} \mathbf{C}$ & Yield $/ \%$ \\
\hline 1 & EtOH & No-MW & 300 & r.t. & 90 \\
2 & EtOH & No-MW & 120 & Reflux & 85 \\
3 & EtOH & MW & 1 & 78 & 68 \\
4 & EtOH & MW & 5 & 78 & 80 \\
5 & EtOH & MW & 10 & 78 & 92 \\
6 & EtOH & MW & 15 & 78 & 92 \\
7 & EtOH & MW & 10 & 70 & 78 \\
8 & EtOH & MW & 10 & 85 & 91 \\
\hline
\end{tabular}


Table 2 The antifungal activity of title compounds in vivo at $100 \mu \mathrm{g} / \mathrm{mL}$

\begin{tabular}{|c|c|c|c|c|}
\hline No. & $\mathbf{R}$ & Stemphylium lycopersici & Fusarium oxysporum & Botrytis cinerea \\
\hline $4 a$ & & 7.14 & 53.89 & 4.44 \\
\hline $4 b$ & & 2.38 & 75.56 & 10.00 \\
\hline $4 c$ & & 2.83 & 38.61 & 12.22 \\
\hline 4d & & 40.18 & 46.67 & 22.22 \\
\hline $4 e$ & & 45.54 & 64.44 & 23.33 \\
\hline $4 f$ & & 39.88 & 13.33 & 21.11 \\
\hline $4 \mathrm{~g}$ & & 26.19 & 16.67 & 29.63 \\
\hline $4 \mathrm{~h}$ & & 63.99 & 31.11 & 20.00 \\
\hline $4 \mathbf{i}$ & & 82.74 & 22.22 & 24.44 \\
\hline 4j & & 69.05 & 4.44 & 21.11 \\
\hline $4 \mathbf{k}$ & & 83.53 & 88.89 & 16.67 \\
\hline 41 & & 62.30 & 77.22 & 20.00 \\
\hline
\end{tabular}


Table 2 continued

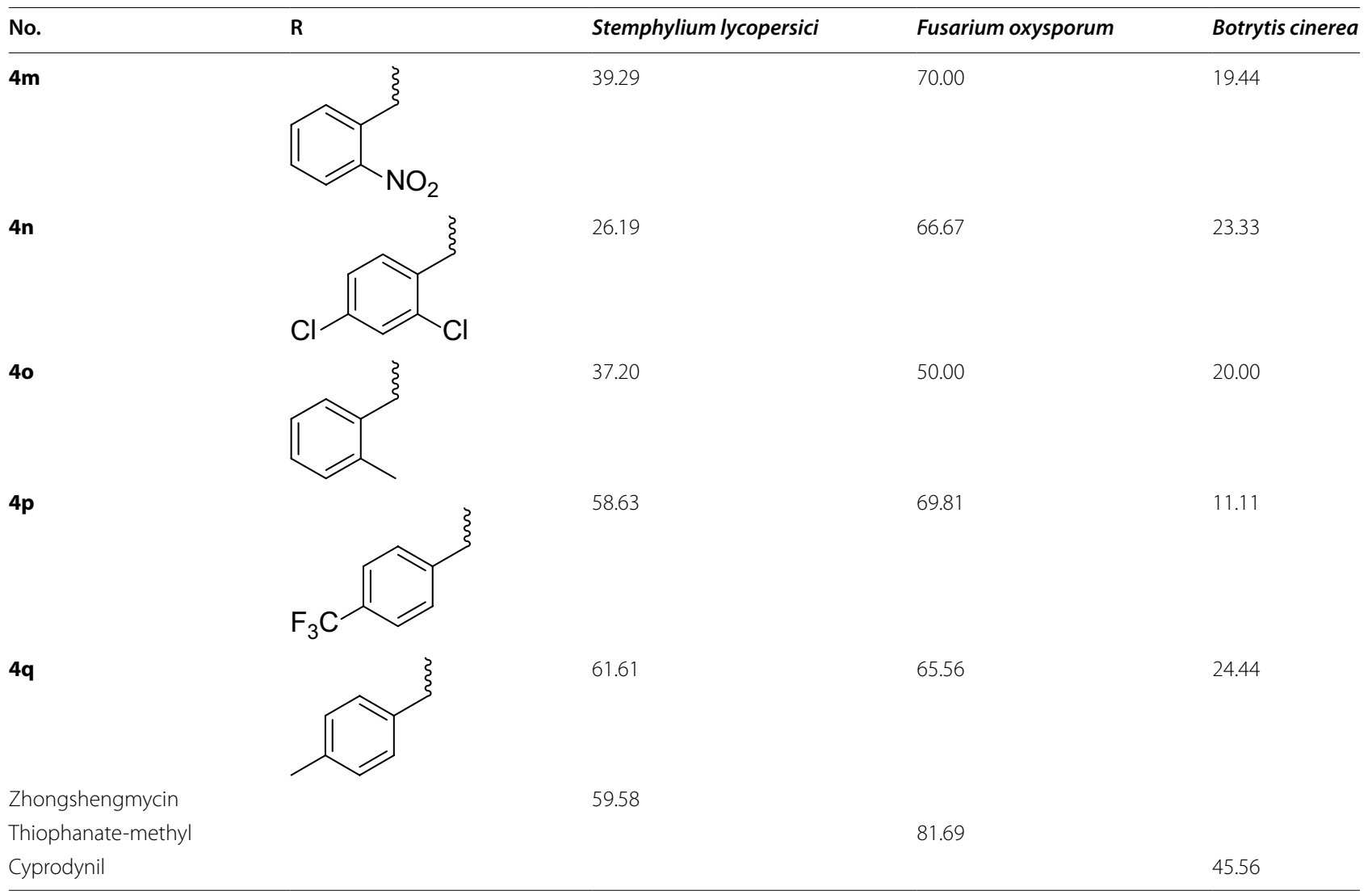

compound $\mathbf{4 i}(82.74 \%)$ and $\mathbf{4 k}(83.53 \%)$ possessed good activity against $\mathrm{SL}$, much better than that of control zhongshengmycin (59.58\%). Among the other, compound $4 h(63.99 \%)$, $4 \mathbf{l}(62.30 \%), 4 p(58.63 \%), 4 q(61.61 \%)$ exhibited good effect against SL, they displayed a comparable level of activity as the control zhongshengmycin. For FO, compound 4k exhibited excellent effect (88.89\%), better than that of thiophanate-methyl(81.69 \%). Meanwhile, compounds 4a, 4b, 4e, 4l, 4m, 4n, 4o, 4p and 4q showed moderate effect against FO with the inhibitory values of $53.89,75.56,64.44,77.22,70.00,66.67,50.00$, $69.81,65.56 \%$ respectively. Unfortunately, most of the compounds had low antifungal activities against Botrytis cinerea.

From Table 2, the preliminary structure and activity relationship (SAR) analysis indicated that compound with electron donating group at para position of benzene ring exhibited significant antifungal activity against SL. For example, compound $4 \mathbf{h}(p-\mathrm{F}), \mathbf{4 l}\left(p-\mathrm{N}\left(\mathrm{CH}_{3}\right)_{2}\right), \mathbf{4} \mathbf{p}(p$ $\left.\mathrm{CF}_{3}\right)$ and $\mathbf{4 q}\left(p-\mathrm{CH}_{3}\right)$ displayed $>50 \%$ inhibitory activities. Also we found that the five-membered ring (Furan ring) held better activity against SL and FO than that of alkyl or aryl group. For the substituted salicylaldehydes, only compound $4 \mathbf{i}$ exhibited excellent antifungal activity against SL. On the other hand, single or poly substituted compounds on the benzene ring both showed good activity against FO.

\section{DFT calculation and SAR}

In order to study their structure-active relationship, we choose a highly active compound $\mathbf{4 k}$ and low activity compound 4c as model compounds; the frontier orbitals and LogP were calculated. The LogP, energy of HOMO and LUMO, total energy and energy gap are listed in Table 3.

Table 3 LogP, total energy, energy gap and frontier orbital energy

\begin{tabular}{lcc}
\hline DFT & $\mathbf{4 c}$ & $\mathbf{4 k}$ \\
\hline$E_{\text {total }} /$ Hartree $^{\mathrm{b}}$ & -1519.50133881 & -1339.28590225 \\
$E_{\text {HOMO }} /$ Hartree & -0.12708 & -0.23503 \\
$E_{\text {LUMO }} /$ Hartree & 0.00908 & -0.07634 \\
$\Delta E^{2} /$ Hartree & 0.13616 & 0.15869 \\
LogP & 1.91 & -0.43 \\
\hline
\end{tabular}

${ }^{\mathrm{a}} \Delta E=E_{\mathrm{LUMO}}-E_{\mathrm{HOMO}}$

b 1 Hartree $=4.35974417 \times 10^{-18}, \mathrm{~J}=27.2113845 \mathrm{eV}$ 
According to the frontier molecular orbital theory, HOMO has the priority to provide electrons, while LUMO can accept electrons firstly $[29,30]$. As we can see from Fig. 1, the LUMO and HOMO are different between the high active compound $\mathbf{4 k}$ and low active compound 4c, especially in the orient of electron transition and energy gap. For the HOMO, the electron of compound $4 \mathbf{k}$ is mainly concentrated on the fused 1,2,4-triazolo[4,3a]pyridine ring and a little on the acyl hydrazine bridge and furan ring, while for the compound $\mathbf{4 c}$, the electron is mainly concentrated on the acyl hydrazine bridge and phenyl ring, but the fused 1,2,4-triazolo[4,3-a] pyridine ring had no electrons. As for the LUMO, The electron of compound $\mathbf{4 k}$ is evenly distributed among the 1,2,4-triazolo[4,3-a]pyridine ring, acyl hydrazone group and furan ring. But the electron of compound $\mathbf{4 c}$ is located on the 1,2,4-triazolo[4,3-a]pyridine ring. The possible reasons of different antifungal activity between the compound $\mathbf{4 c}$ and $\mathbf{4 k}$ is electron transition direction and energy gap. From Fig. 1, we assumed that the compound with higher energy gap exhibited higher antifungal activity. Also the 1,2,4-triazolo[4,3-a]pyridine ring is important for the higher active compound. The other impact fact is LogP. From Table 1, the $\log \mathrm{P}$ is different between the two compounds.

The optimized structures of the compound $\mathbf{4 c}$ and 4k are presented in Fig. 2. From Fig. 2, we can find that the orientations of amide groups are different. As we known, the conformation of compound is important for the biological activity due to the bind mode between the receptor and acceptor. So we speculate that the conformation of highly active compound is perpendicular between the 1,2,4-triazolo[4,3-a]pyridine ring and the aromatic ring. Otherwise, when the conformation of low active compound, the aromatic ring is parallel with the 1,2,4-triazolo[4,3-a]pyridine ring. These important clues will be helpful in the design of more potent compounds in the future.

\section{Methods}

\section{Instruments}

All the chemical reagents are analytical grade or prepared by our lab. Melting points were measured using an X-4 apparatus and were uncorrected. ${ }^{1} \mathrm{H}$ NMR spectra were recorded on a Bruker Avance $500 \mathrm{MHz}$ spectrometer using DMSO- $\mathrm{d}_{6}$ as solvent. ${ }^{13} \mathrm{C}$ NMR and ${ }^{19} \mathrm{~F}$ NMR spectra were recorded on a Bruker Avance $600 \mathrm{MHz}$ spectrometer using DMSO- $\mathrm{d}_{6}$ as solvent. Mass spectra were determined on a Thermo Finnigan LCQ Advantage LC/ mass detector instrument. Elemental analysis data of title compounds were collected by a Perkin-Elmer 240C analyzer. CEM Discover Focused Synthesizer was used to carry out the microwave reaction $(600 \mathrm{~W}, 2450 \mathrm{MHz})$.

\section{Synthesis}

The key intermediate 1, 2, 3 are synthesized according to our previous work [28]. The title compounds $\mathbf{4 a - 4 q}$ was synthesized from the intermediate $\mathbf{3}$ and different aldehydes or ketones in the solution of ethanol at the condition of microwave $\left(150 \mathrm{w}, 78{ }^{\circ} \mathrm{C}, 200 \mathrm{psi}, 10 \mathrm{~min}\right)$. All the other compounds are synthesized according to the procedure (Scheme 2).

8-Chloro-N'-(1-phenylethylidene)-[1,2,4]triazolo[4,3a]pyridine-3-carbohydrazide (4a) white yellow crystal,

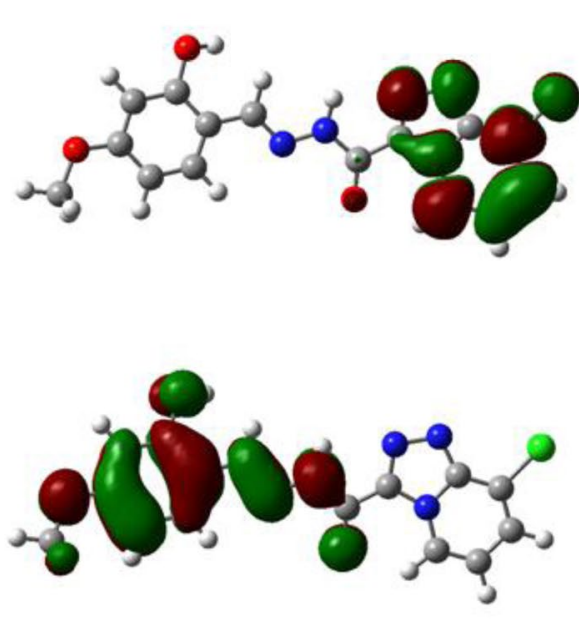

4c

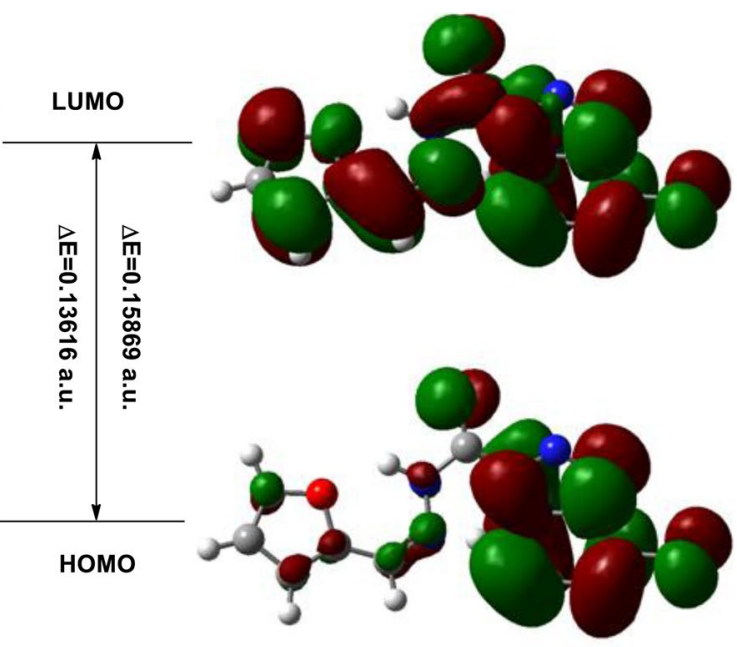

$4 k$

Fig. 1 Frontier molecular orbitals of compound $\mathbf{4 c}$ and $\mathbf{4 k}$ 


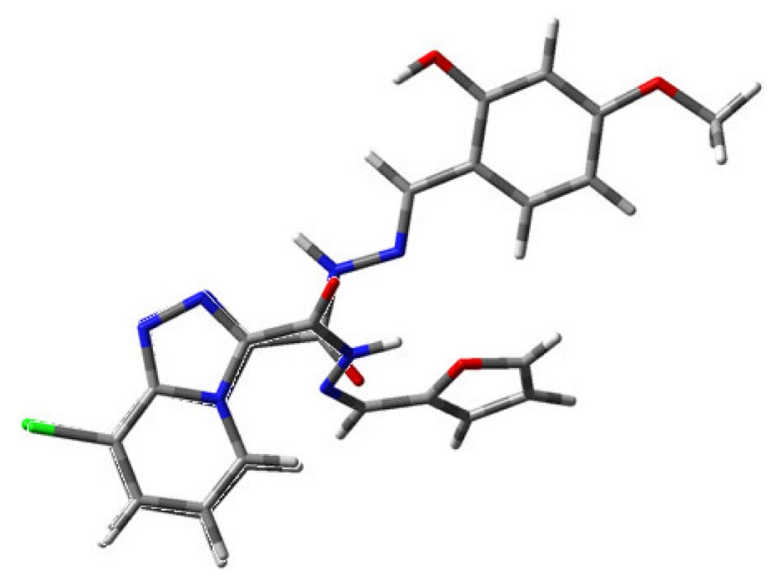

Fig. 2 Overlay of energy-minimized structures of $\mathbf{4 c}$ and $\mathbf{4 k}$

yield $82 \%$, m.p. > $300{ }^{\circ} \mathrm{C}$; FT-IR $\left(\mathrm{KBr}, \mathrm{cm}^{-1}\right): v 3416$, 3216, 3128, 1690, 1662, 1539, 1478, 1382, 1342, 1258, 1217, 1088, 948, 860, 794; ${ }^{1} \mathrm{H}$ NMR (DMSO-d ${ }_{6}$, $500 \mathrm{MHz}), \delta: 2.51\left(\mathrm{~s}, 3 \mathrm{H}, \mathrm{CH}_{3}\right), 7.08(\mathrm{t}, J=7.0 \mathrm{~Hz}, 1 \mathrm{H}$, Py), 7.46(s, 3H, Ar), 7.56(d, $J=7.5 \mathrm{~Hz}, 1 \mathrm{H}, \mathrm{Py}), 7.94(\mathrm{~s}$, $2 \mathrm{H}, \mathrm{Ar}), 9.38(\mathrm{~d}, J=7.0 \mathrm{~Hz}, 1 \mathrm{H}, \mathrm{Py}), 10.35(\mathrm{~s}, 1 \mathrm{H}, \mathrm{NH})$; ${ }^{13} \mathrm{C}$ NMR $\left(150 \mathrm{MHz}\right.$, DMSO-d $\left.\mathrm{d}_{6}\right) \delta 15.19,116.52,120.83$, 125.56, 125.80, 127.11, 128.95, 129.26, 129.39, 138.06, 139.91, 141.37, 149.04, 156.91, 168.78; MS (ESI), m/z: $314(\mathrm{M}+1)^{+}$. Elemental anal. For $\mathrm{C}_{15} \mathrm{H}_{12} \mathrm{ClN}_{5} \mathrm{O}(\%)$, calculated: $\mathrm{C}, 57.42 ; \mathrm{H}, 3.86 ; \mathrm{N}, 22.32$; found: $\mathrm{C}, 57.65 ; \mathrm{H}$, 3.76; N, 22.51.

8-Chloro- $N^{\prime}$-ethylidene-[1,2,4]triazolo[4,3-a]pyridine3-carbohydrazide (4b) white yellow crystal, yield $92 \%$, m.p. > $300{ }^{\circ} \mathrm{C}$; FT-IR $\left(\mathrm{KBr}, \mathrm{cm}^{-1}\right)$ : v 3219, 3116, 2995, 1724, 1686, 1578, 1491, 1239, 1109, 1039, 855, 794, 688, $535 ;{ }^{1} \mathrm{H}$ NMR (DMSO-d $\left.{ }_{6}, 500 \mathrm{MHz}\right), \delta: 1.95\left(\mathrm{~s}, 3 \mathrm{H}, \mathrm{CH}_{3}\right)$, $7.24(\mathrm{t}, J=7.2 \mathrm{~Hz}, 1 \mathrm{H}, \mathrm{Py}), 7.82(\mathrm{~d}, J=7.3 \mathrm{~Hz}, 1 \mathrm{H}, \mathrm{Py})$, $9.11(\mathrm{~d}, J=7.0 \mathrm{~Hz}, 1 \mathrm{H}, \mathrm{Py}), 10.03(\mathrm{~s}, 1 \mathrm{H}, \mathrm{CH}), 11.02(\mathrm{~s}, 1 \mathrm{H}$, $\mathrm{NH}) ;{ }^{13} \mathrm{C}$ NMR (150 MHz, DMSO-d 6 ) $\delta 21.04,116.52$, 120.82, 125.55, 129.25, 139.90, 149.03, 156.90, 168.78; MS (ESI), m/z: $238(\mathrm{M}+1)^{+}$. Elemental anal. For $\mathrm{C}_{9} \mathrm{H}_{8} \mathrm{ClN}_{5} \mathrm{O}$ (\%), calculated: $\mathrm{C}, 45.49 ; \mathrm{H}, 3.39 ; \mathrm{N}, 29.47$; found: $\mathrm{C}$, $45.55 ; \mathrm{H}, 3.21 ; \mathrm{N}, 29.65$.

8-Chloro- $N^{\prime}$-(2-hydroxy-4-methoxybenzylidene)-[1,2,4] triazolo[4,3-a]pyridine-3-carbohydrazide (4c) white yellow crystal, yield $90 \%$, m.p. > $300{ }^{\circ} \mathrm{C}$; FT-IR $(\mathrm{KBr}$, $\left.\mathrm{cm}^{-1}\right): v 3500,3141,3083,1709,1677,1631,1606,1508$, 1457, 1251, 1230, 1204, 1170, 1092, 1022, 965, 832, 742; ${ }^{1} \mathrm{H}$ NMR (DMSO-d 6 , $\left.500 \mathrm{MHz}\right), \delta: 3.79\left(\mathrm{~s}, 3 \mathrm{H}, \mathrm{OCH}_{3}\right.$ ), $6.55(\mathrm{~d}, J=9.0 \mathrm{~Hz}, 1 \mathrm{H}, \mathrm{Ar}), 7.25(\mathrm{t}, J=7.2 \mathrm{~Hz}, 1 \mathrm{H}, \mathrm{Py})$, $7.43(\mathrm{~d}, J=8.5 \mathrm{~Hz}, 1 \mathrm{H}, \mathrm{Py}), 7.83(\mathrm{~d}, J=7.3 \mathrm{~Hz}, 1 \mathrm{H}, \mathrm{Ar})$, 8.74(s, $1 \mathrm{H}, A r), 9.22(\mathrm{~d}, J=7.0 \mathrm{~Hz}, 1 \mathrm{H}, \mathrm{Py}), 11.49(\mathrm{~s}$, $1 \mathrm{H}, \mathrm{CH}), 11.97(\mathrm{~s}, 1 \mathrm{H}, \mathrm{NH}), 13.01(\mathrm{~s}, 1 \mathrm{H}, \mathrm{OH}) ;{ }^{13} \mathrm{C}$ NMR $\left(150 \mathrm{MHz}\right.$, DMSO-d $\left.{ }_{6}\right) \delta 55.84,101.69,107.15$, $112.19,116.42,120.78,125.92,129.38,131.79,140.42$,
149.14, 150.93, 153.82, 160.03, 162.86; MS (ESI), m/z: $346(\mathrm{M}+1)^{+}$. Elemental anal. For $\mathrm{C}_{15} \mathrm{H}_{12} \mathrm{ClN}_{5} \mathrm{O}_{3}(\%)$, calculated: C, $52.11 ; \mathrm{H}, 3.50 ; \mathrm{N}, 20.26$; found: $\mathrm{C}, 51.98 ; \mathrm{H}$, 3.44; N, 20.43.

8-Chloro-N'-(2-hydroxybenzylidene)-[1,2,4] triazolo[4,3-a]pyridine-3-carbohydrazide (4d) white yellow crystal, yield $92 \%$, m.p. > $300{ }^{\circ} \mathrm{C}$; FT-IR $\left(\mathrm{KBr}, \mathrm{cm}^{-1}\right)$ : v 3178, 3143, 3052, 1667, 1619, 1549, 1486, 1445, 1354, $1271,1271,1238,1220,1096,1039,954,848,762 ;{ }^{1} \mathrm{H}$ NMR (DMSO-d $\left.\mathrm{d}_{6}, 500 \mathrm{MHz}\right), \delta: 6.92-6.96(\mathrm{~m}, 2 \mathrm{H}, \mathrm{Ar})$, 7.23-7.33(m, 2H, Ar), 7.54(t, $J=7.2 \mathrm{~Hz}, 1 \mathrm{H}, \mathrm{Py}), 7.82(\mathrm{~s}$, $1 \mathrm{H}, \mathrm{CH}), 8.28(\mathrm{~d}, J=7.3 \mathrm{~Hz}, 1 \mathrm{H}, \mathrm{Py}), 9.21(\mathrm{~d}, J=7.0 \mathrm{~Hz}$, $1 \mathrm{H}, \mathrm{Py}), 11.13(\mathrm{~s}, 1 \mathrm{H}, \mathrm{NH}), 13.10(\mathrm{~s}, 1 \mathrm{H}, \mathrm{OH}) ;{ }^{13} \mathrm{C}$ NMR $\left(150 \mathrm{MHz}, \mathrm{DMSO}-\mathrm{d}_{6}\right) \delta 116.48,116.98,119.15,119.94$, $120.79,125.93,129.43,130.02,132.25,140.40,149.17$, 150.41, 154.08, 159.03; MS (ESI), m/z: $316(\mathrm{M}+1)^{+}$. Elemental anal. For $\mathrm{C}_{14} \mathrm{H}_{10} \mathrm{ClN}_{5} \mathrm{O}_{2}$ (\%), calculated: $\mathrm{C}$, 53.26; $\mathrm{H}, 3.19$; N, 22.18; found: C, 53.35; H, 3.22; N, 22.41 .

8-Chloro-N'-(3-nitrobenzylidene)-[1,2,4]triazolo[4,3a]pyridine-3-carbohydrazide (4e) white yellow crystal, yield $94 \%$, m.p. $>300{ }^{\circ} \mathrm{C}$; FT-IR $\left(\mathrm{KBr}, \mathrm{cm}^{-1}\right)$ : v 3322, 3158, 1683, 1620, 1533, 1486, 1451, 1353, 1275, 1214, 1146, 1078, 959, 897, 853, 788, 736; ${ }^{1} \mathrm{H}$ NMR (DMSO-d , , $500 \mathrm{MHz}), \delta: 7.27(\mathrm{t}, J=7.0 \mathrm{~Hz}, 1 \mathrm{H}, \mathrm{Py}), 7.73(\mathrm{t}, J=7.8 \mathrm{~Hz}$, $1 \mathrm{H}, \mathrm{Ar}), 7.84-7.87(\mathrm{~m}, 2 \mathrm{H}, \mathrm{Py} \& \mathrm{Ar}), 8.10 \sim 8.17(\mathrm{~m}, 2 \mathrm{H}$, Ar), 9.08(s, $1 \mathrm{H}, \mathrm{CH}), 9.21(\mathrm{~d}, J=6.8 \mathrm{~Hz}, 1 \mathrm{H}, \mathrm{Py}), 13.21(\mathrm{~s}$, $1 \mathrm{H}, \mathrm{NH}) ;{ }^{13} \mathrm{C}$ NMR $\left(150 \mathrm{MHz}, \mathrm{DMSO}-\mathrm{d}_{6}\right) \delta 116.58$, $120.83,121.57,125.08,125.87,129.48,131.09,134.06$, 136.39, 140.48, 147.71, 148.75, 154.48, 162.17; MS (ESI), $\mathrm{m} / \mathrm{z}: 345(\mathrm{M}+1)^{+}$. Elemental anal. For $\mathrm{C}_{14} \mathrm{H}_{9} \mathrm{ClN}_{6} \mathrm{O}_{3}(\%)$, calculated: $\mathrm{C}, 48.78 ; \mathrm{H}, 2.63 ; \mathrm{N}, 24.38$; found: $\mathrm{C}, 48.86 ; \mathrm{H}$, $2.76 ; \mathrm{N}, 24.99$.

8-Chloro-N'-(5-chloro-2-hydroxybenzylidene)-[1,2,4] triazolo[4,3-a]pyridine-3-carbohydrazide (4f) white yellow crystal, yield $88 \%$, m.p. $>300{ }^{\circ} \mathrm{C}$; FT-IR $\left(\mathrm{KBr}, \mathrm{cm}^{-1}\right)$ : v 3246, 3135, 1676, 1618, 1521, 1477, 1458, 1342, 1267, 1211, 1184, 1145, 1084, 846, 724; ${ }^{1} \mathrm{H}$ NMR (DMSO$\left.\mathrm{d}_{6}, 500 \mathrm{MHz}\right), \delta: 6.98(\mathrm{~d}, J=8.7 \mathrm{~Hz}, 1 \mathrm{H}, \mathrm{Ar}), 7.28(\mathrm{t}$, $J=7.2 \mathrm{~Hz}, 1 \mathrm{H}, \mathrm{Py}), 7.35(\mathrm{~d}, J=8.5 \mathrm{~Hz}, 1 \mathrm{H}, \mathrm{Py}), 7.68(\mathrm{~s}$, $1 \mathrm{H}, \mathrm{Ar}), 7.93(\mathrm{~d}, J=7.4 \mathrm{~Hz}, 1 \mathrm{H}, \mathrm{Ar}), 8.82(\mathrm{~s}, 1 \mathrm{H}, \mathrm{CH})$, $9.21(\mathrm{~d}, J=6.9 \mathrm{~Hz}, 1 \mathrm{H}, \mathrm{Py}), 11.05(\mathrm{~s}, 1 \mathrm{H}, \mathrm{NH}), 13.16(\mathrm{~s}, 1 \mathrm{H}$, $\mathrm{OH}) ;{ }^{13} \mathrm{C}$ NMR (150 MHz, DMSO-d 6 ) 8116.51, 118.80, $120.80,121.29,123.54,125.92,127.86,129.45,131.62$, 140.41, 147.80, 149.18, 154.25, 156.59; MS (ESI), m/z: $350(\mathrm{M}+1)^{+}$. Elemental anal. For $\mathrm{C}_{14} \mathrm{H}_{9} \mathrm{Cl}_{2} \mathrm{~N}_{5} \mathrm{O}_{2}$ (\%), calculated: $\mathrm{C}, 48.02 ; \mathrm{H}, 2.59 ; \mathrm{N}, 20.00$; found: $\mathrm{C}, 47.80 ; \mathrm{H}$, $2.75 ; \mathrm{N}, 20.21$.

$N^{\prime}$-(5-bromo-2-hydroxybenzylidene)-8-chloro- $[1,2,4]$ triazolo[4,3-a]pyridine-3-carbohydrazide (4g) white yellow crystal, yield $90 \%$, m.p. $>300{ }^{\circ} \mathrm{C}$; FT-IR $\left(\mathrm{KBr}, \mathrm{cm}^{-1}\right)$ : v 3249, 3134, 1674, 1619, 1613, 1519, 1473, 1455, 1341, 1266, 1210, 1182, 1077, 958, 846, 742; ${ }^{1} \mathrm{H}$ NMR (DMSO$\left.\mathrm{d}_{6}, 500 \mathrm{MHz}\right), \delta: 6.92(\mathrm{~d}, J=8.7 \mathrm{~Hz}, 1 \mathrm{H}, \mathrm{Ar}), 7.27(\mathrm{t}$, 
$J=7.2 \mathrm{~Hz}, 1 \mathrm{H}, \mathrm{Py}), 7.45(\mathrm{~d}, J=8.5 \mathrm{~Hz}, 1 \mathrm{H}, \mathrm{Py}), 7.80(\mathrm{~s}$, $1 \mathrm{H}, \mathrm{Ar}), 7.84(\mathrm{~d}, J=7.4 \mathrm{~Hz}, 1 \mathrm{H}, \mathrm{Ar}), 8.82(\mathrm{~s}, 1 \mathrm{H}, \mathrm{CH})$, $9.21(\mathrm{~d}, J=6.9 \mathrm{~Hz}, 1 \mathrm{H}, \mathrm{Py}), 11.10(\mathrm{~s}, 1 \mathrm{H}, \mathrm{NH}), 13.15(\mathrm{~s}, 1 \mathrm{H}$, $\mathrm{OH}) ;{ }^{13} \mathrm{C}$ NMR (150 MHz, DMSO-d $\left.{ }_{6}\right) \delta 116.58,120.83$, $121.57,125.08,125.87,129.48,131.09,134.06,136.39$, 140.48, 147.71, 148.75, 149.21, 154.48; MS (ESI), m/z: $395(\mathrm{M}+1)^{+}$. Elemental anal. For $\mathrm{C}_{14} \mathrm{H}_{9} \mathrm{BrClN}_{5} \mathrm{O}_{2}(\%)$, calculated: $\mathrm{C}, 42.61 ; \mathrm{H}, 2.30 ; \mathrm{N}, 17.75$; found: $\mathrm{C}, 42.45 ; \mathrm{H}$, 2.25; N, 17.71.

8-Chloro-N'-(1-(4-fluorophenyl)ethylidene)-[1,2,4] triazolo[4,3-a]pyridine-3-carbohydrazide (4h) white yellow crystal, yield $93 \%$, m.p. $>300{ }^{\circ} \mathrm{C}$; FT-IR $\left(\mathrm{KBr}, \mathrm{cm}^{-1}\right)$ : v $3345,3223,3137,1698,1662,1538,1504,1497,1382$, 1342, 1217, 1158, 1089, 949, 858, 794, 742; ${ }^{1} \mathrm{H}$ NMR (DMSO-d $\left.{ }_{6}, 500 \mathrm{MHz}\right), \delta: 2.47\left(\mathrm{~s}, 3 \mathrm{H}, \mathrm{CH}_{3}\right), 7.05-7.13(\mathrm{~m}$, $3 \mathrm{H}, \mathrm{Ar}$ and Py), 7.54(t, $J=7.2 \mathrm{~Hz}, 1 \mathrm{H}, \mathrm{Py}), 7.90-7.93(\mathrm{~m}$, $2 \mathrm{H}, \mathrm{Ar}), 9.35(\mathrm{~d}, J=6.9 \mathrm{~Hz}, 1 \mathrm{H}, \mathrm{Py}), 10.31(\mathrm{~s}, 1 \mathrm{H}, \mathrm{NH})$; ${ }^{13} \mathrm{C}$ NMR $\left(150 \mathrm{MHz}, \mathrm{DMSO}-\mathrm{d}_{6}\right) \delta 15.23,115.80,115.93$, $116.51,120.83,125.55,125.78,129.25,129.40,139.91$, 149.04, 156.91, 168.78; ${ }^{19} \mathrm{~F}$ NMR (564 MHz, DMSO-d ${ }_{6}$ ) $\delta$-111.38; MS (ESI), m/z: 332(M+1) ${ }^{+}$. Elemental anal. For $\mathrm{C}_{15} \mathrm{H}_{11} \mathrm{ClFN}_{5} \mathrm{O}$ (\%), calculated: $\mathrm{C}, 54.31 ; \mathrm{H}, 3.34 ; \mathrm{N}$, 21.11; found: C, 54.18; $\mathrm{H}, 3.52 ; \mathrm{N}, 21.31$.

8-Chloro- $N^{\prime}$ - (4-(diethylamino) - 2 hydroxybenzylidene)-[1,2,4]triazolo[4,3-a]pyridine3-carbohydrazide (4i) white yellow crystal, yield $92 \%$, m.p. $>300{ }^{\circ} \mathrm{C}$; FT-IR $\left(\mathrm{KBr}, \mathrm{cm}^{-1}\right): v$ 3212, 2970, 2931, 1676, 1629, 1596, 1518, 1488, 1350, 1247, 1132, 1078, 1040, 850, 758, 738; ${ }^{1} \mathrm{H}$ NMR (DMSO-d ${ }_{6}, 500 \mathrm{MHz}$ ), $\delta: 1.18-1.25\left(\mathrm{~m}, 6 \mathrm{H}, 2 \mathrm{CH}_{3}\right), 3.36-3.42\left(\mathrm{~m}, 4 \mathrm{H}, 2 \mathrm{CH}_{2}\right)$, 6.62(m, 2H, Ar), 7.03-7.10(m, 2H, Py and Ar), 7.52(d, $J=7.2 \mathrm{~Hz}, 1 \mathrm{H}, \mathrm{Py}), 8.25(\mathrm{~s}, 1 \mathrm{H}, \mathrm{Ar}), 8.45(\mathrm{~s}, 1 \mathrm{H}, \mathrm{CH})$, 9.34(d, $J=7.1 \mathrm{~Hz}, 1 \mathrm{H}, \mathrm{Py}), 10.22(\mathrm{~s}, 1 \mathrm{H}, \mathrm{NH}) ;{ }^{13} \mathrm{C} \mathrm{NMR}$ $\left(150 \mathrm{MHz}, \mathrm{DMSO}-\mathrm{d}_{6}\right) \delta 13.02,44.30,97.93,104.31$, $106.31,116.30,120.75,125.92,129.25,132.37,140.55$, 149.06, 150.92, 152.06, 153.35, 160.35; MS (ESI), m/z: $387(\mathrm{M}+1)^{+}$. Elemental anal. For $\mathrm{C}_{18} \mathrm{H}_{19} \mathrm{ClN}_{6} \mathrm{O}_{2}(\%)$, calculated: $\mathrm{C}, 55.89 ; \mathrm{H}, 4.95 ; \mathrm{N}, 21.73$; found: $\mathrm{C}, 55.99 ; \mathrm{H}$, 4.76; N, 21.69.

8-Chloro- $N^{\prime}$-(4-(dimethylamino)benzylidene)-[1,2,4] triazolo[4,3-a]pyridine-3-carbohydrazide (4j) white yellow crystal, yield $90 \%$, m.p. $280-281{ }^{\circ} \mathrm{C}$; FT-IR (KBr, $\left.\mathrm{cm}^{-1}\right): v 3487,1674,1596,1525,1466,1367,1255$, 1189, 1087, 809, 740; ${ }^{1} \mathrm{H}$ NMR (DMSO-d 6 , $500 \mathrm{MHz}$ ), $\delta: 2.99\left(\mathrm{~s}, 6 \mathrm{H}, 2 \mathrm{CH}_{3}\right), 6.78(\mathrm{~d}, J=8.8 \mathrm{~Hz}, 2 \mathrm{H}, \mathrm{Ar}), 7.24(\mathrm{t}$, $J=7.2 \mathrm{~Hz}, 1 \mathrm{H}, \mathrm{Py}), 7.54(\mathrm{~d}, J=8.7 \mathrm{~Hz}, 1 \mathrm{H}, \mathrm{Ar}), 7.81(\mathrm{~d}$, $J=7.2 \mathrm{~Hz}, 1 \mathrm{H}, \mathrm{Py}), 8.49(\mathrm{~s}, 1 \mathrm{H}, \mathrm{CH}), 9.21(\mathrm{~d}, J=6.9 \mathrm{~Hz}$, $1 \mathrm{H}, \mathrm{Py}), 12.50(\mathrm{~s}, 1 \mathrm{H}, \mathrm{NH}) ;{ }^{13} \mathrm{C}$ NMR $(150 \mathrm{MHz}, \mathrm{DMSO}-$ $\left.\mathrm{d}_{6}\right) \delta 40.22,112,25,116.26,120.75,121.72,125.86,129.18$, 138.76, 140.77, 149.03, 150.89, 152.21, 153.69; MS (ESI), $\mathrm{m} / \mathrm{z}: 343(\mathrm{M}+1)^{+}$. Elemental anal. For $\mathrm{C}_{16} \mathrm{H}_{15} \mathrm{ClN}_{6} \mathrm{O}(\%)$, calculated: $\mathrm{C}, 56.06 ; \mathrm{H}, 4.41 ; \mathrm{N}, 24.52$; found: $\mathrm{C}, 55.89 ; \mathrm{H}$, $4.47 ; \mathrm{N}, 24.46$.
8-Chloro- $N^{\prime}$-(furan-2-ylmethylene)-[1,2,4]triazolo[4,3a]pyridine-3-carbohydrazide (4k) white yellow crystal, yield $91 \%$, m.p. $278-279{ }^{\circ} \mathrm{C}$; FT-IR $\left(\mathrm{KBr}, \mathrm{cm}^{-1}\right)$ : v 3269, 3160, 3065, 1666, 1626, 1541, 1479, 1349, 1298, 1220, 1156, 1081, 1012, 935, 851, 803, 732; ${ }^{1}$ H NMR (DMSO-d ${ }_{6}$, $500 \mathrm{MHz}$ ), $\delta: 6.93(\mathrm{~d}, 2 \mathrm{H}$, Furan), $7.07(\mathrm{t}, J=6.9 \mathrm{~Hz}, 1 \mathrm{H}$, Py), 7.54-7.57(m, 2H, Py and Furan), 8.32(s, $1 \mathrm{H}, \mathrm{CH})$, 9.33(d, $J=6.9 \mathrm{~Hz}, 1 \mathrm{H}, \mathrm{Py}), 11.20(\mathrm{~s}, 1 \mathrm{H}, \mathrm{NH}) ;{ }^{13} \mathrm{C} \mathrm{NMR}$ $\left(150 \mathrm{MHz}, \mathrm{DMSO}-\mathrm{d}_{6}\right) \delta 112.84,114.79,116.44,120.77$, 125.91, 129.36, 139.57, 140.54, 146.06, 149.13, 149.74, 154.12; MS (ESI), m/z: $290(\mathrm{M}+1)^{+}$. Elemental anal. For $\mathrm{C}_{12} \mathrm{H}_{8} \mathrm{ClN}_{5} \mathrm{O}_{2}$ (\%), calculated: $\mathrm{C}, 49.75 ; \mathrm{H}, 2.78 ; \mathrm{N}, 24.18$; found: $\mathrm{C}, 49.68 ; \mathrm{H}, 2.76 ; \mathrm{N}, 24.44$.

8-Chloro- $N^{\prime}-(3,4,5$-trimethoxybenzylidene)-[1,2,4] triazolo[4,3-a]pyridine-3-carbohydrazide (4l) white yellow crystal, yield $88 \%$, m.p. > $300{ }^{\circ} \mathrm{C}$; FT-IR $\left(\mathrm{KBr}, \mathrm{cm}^{-1}\right)$ : v 3139, 2994, 1657, 1629, 1569, 1519, 1346, 1246, 1226, 1066, 913, 850, 739; ${ }^{1} \mathrm{H}$ NMR (DMSO-d 6 , $500 \mathrm{MHz}$ ), $\delta$ : 3.96(s, 9H, $\left.3 \mathrm{OCH}_{3}\right), 7.04(\mathrm{~s}, 2 \mathrm{H}, \mathrm{Ar}), 7.28(\mathrm{t}, J=7.0 \mathrm{~Hz}$, $1 \mathrm{H}, \mathrm{Py}), 7.84(\mathrm{~d}, J=6.9 \mathrm{~Hz}, 1 \mathrm{H}, \mathrm{Py}), 8.55(\mathrm{~s}, 1 \mathrm{H}, \mathrm{CH})$, 9.21(d, $J=7.2 \mathrm{~Hz}, 1 \mathrm{H}, \mathrm{Py}), 12.83(\mathrm{~s}, 1 \mathrm{H}, \mathrm{NH}) ;{ }^{13} \mathrm{C} \mathrm{NMR}$ $\left(150 \mathrm{MHz}, \mathrm{DMSO}-\mathrm{d}_{6}\right) \delta 56.46,60.62,104.95,116.44$, $120.80,125.85,129.34,130.05,139.98,139.98,140.60$, 149.13, 153.70, 154.16; MS (ESI), m/z: $391(\mathrm{M}+1)^{+}$. Elemental anal. For $\mathrm{C}_{17} \mathrm{H}_{16} \mathrm{ClN}_{5} \mathrm{O}_{4}$ (\%), calculated: $\mathrm{C}$, 52.38; $\mathrm{H}, 4.14 ; \mathrm{N}, 17.97$; found: $\mathrm{C}, 52.51 ; \mathrm{H}, 4.28 ; \mathrm{N}, 8.21$.

8-Chloro-N'-(2-nitrobenzylidene)-[1,2,4]triazolo[4,3a]pyridine-3-carbohydrazide (4m) white yellow crystal, yield $90 \%$, m.p. $>300{ }^{\circ} \mathrm{C}$; FT-IR $\left(\mathrm{KBr}, \mathrm{cm}^{-1}\right)$ : v 3290, 1679, 1628, 1584, 1536, 1470, 1452, 1361, 1226, 1211, 1153, 1094, 1049, 914, 843, 743; ${ }^{1} \mathrm{H}$ NMR (DMSO-d ${ }_{6}$, $500 \mathrm{MHz}), \delta: 7.27(\mathrm{t}, J=7.0 \mathrm{~Hz}, 1 \mathrm{H}, \mathrm{Py}), 7.72(\mathrm{t}, J=7.8 \mathrm{~Hz}$, $1 \mathrm{H}, \mathrm{Py}), 7.84-7.87(\mathrm{~m}, 2 \mathrm{H}, \mathrm{Ar}), 8.10-8.17(\mathrm{~m}, 2 \mathrm{H}, \mathrm{Ar})$, 9.08(s, $1 \mathrm{H}, \mathrm{CH}), 9.21(\mathrm{~d}, J=6.8 \mathrm{~Hz}, 1 \mathrm{H}, \mathrm{Py}), 12.21(\mathrm{~s}, 1 \mathrm{H}$, $\mathrm{NH}) ;{ }^{13} \mathrm{C}$ NMR $\left(150 \mathrm{MHz}, \mathrm{DMSO}-\mathrm{d}_{6}\right) \delta 116.52,120.81$, $125.19,125.89,128.63,128.98,129.46,131.50,134.29$, 140.51, 145.52, 148.85, 149.19, 154.54; MS (ESI), m/z: $345(\mathrm{M}+1)^{+}$. Elemental anal. For $\mathrm{C}_{14} \mathrm{H}_{9} \mathrm{ClN}_{6} \mathrm{O}_{3}$ (\%), calculated: C, 48.78; H, 2.63; N, 24.38; found: $\mathrm{C}, 48.95 ; \mathrm{H}$, $2.45 ; \mathrm{N}, 24.43$.

8-Chloro- $N^{\prime}$-(2,4-dichlorobenzylidene)-[1,2,4] triazolo[4,3-a]pyridine-3-carbohydrazide (4n) white yellow crystal, yield $91 \%$, m.p. $>300{ }^{\circ} \mathrm{C}$; FT-IR $\left(\mathrm{KBr}, \mathrm{cm}^{-1}\right)$ : v 3323, 3158, 3082, 1684, 1619, 1532, 1487, 1451, 1353, 1214, 1146, 1078, 1048, 958, 897, 853, 788, 735, 694; ${ }^{1} \mathrm{H}$ NMR (DMSO-d $\left.{ }_{6}, 500 \mathrm{MHz}\right), \delta: 7.27(\mathrm{t}, J=7.0 \mathrm{~Hz}, 1 \mathrm{H}$, Py), 7.53(d, $J=8.3 \mathrm{~Hz}, 1 \mathrm{H}, \mathrm{Ar}), 7.74(\mathrm{~s}, 1 \mathrm{H}, \mathrm{Ar}), 7.85(\mathrm{~d}$, $J=7.2 \mathrm{~Hz}, 1 \mathrm{H}, \mathrm{Py}), 8.03(\mathrm{~d}, J=8.5 \mathrm{~Hz}, 1 \mathrm{H}, \mathrm{Ar}), 9.05(\mathrm{~s}$, $1 \mathrm{H}, \mathrm{CH}), 9.21(\mathrm{~d}, J=6.9 \mathrm{~Hz}, 1 \mathrm{H}, \mathrm{Py}), 12.13(\mathrm{~s}, 1 \mathrm{H}, \mathrm{NH})$; ${ }^{13} \mathrm{C}$ NMR (150 MHz, DMSO-d $\left.\mathrm{d}_{6}\right) \delta 116.55,120.83$, $125.88,128.58,128.64,129.46,129.97,131.09,134.72$, 135.92, 140.49, 145.14, 149.20, 154.40; MS (ESI), m/z: $369(\mathrm{M}+1)^{+}$. Elemental anal. For $\mathrm{C}_{14} \mathrm{H}_{8} \mathrm{Cl}_{3} \mathrm{~N}_{5} \mathrm{O}$ (\%), 
calculated: C, 45.62; H, 2.19; N, 19.00; found: C, 45.65; H, 2.33; N, 19.21 .

8-Chloro-N'-(2-methylbenzylidene)-[1,2,4]triazolo[4,3a]pyridine-3-carbohydrazide (4o) white yellow crystal, yield $86 \%$, m.p. $>300{ }^{\circ} \mathrm{C}$; FT-IR $\left(\mathrm{KBr}, \mathrm{cm}^{-1}\right): v 3222$, 3060, 1680, 1598, 1548, 1523, 1488, 1454, 1359, 1234, 1222, 1112, 1085, 1039, 949, 849, 784, 742, 693; ${ }^{1} \mathrm{H}$ NMR (DMSO- $\left.\mathrm{d}_{6}, 500 \mathrm{MHz}\right), \delta: 2.37\left(\mathrm{~s}, 3 \mathrm{H}, \mathrm{CH}_{3}\right), 7.24-7.28(\mathrm{~m}$, $1 \mathrm{H}, \mathrm{Ar}), 7.37(\mathrm{t}, J=7.5 \mathrm{~Hz}, 1 \mathrm{H}, \mathrm{Py}), 7.53(\mathrm{~d}, J=7.5 \mathrm{~Hz}, 1 \mathrm{H}$, Ar), 7.57(s, $1 \mathrm{H}, \mathrm{Ar}), 7.73(\mathrm{~d}, J=7.2 \mathrm{~Hz}, 1 \mathrm{H}, \mathrm{Py}), 7.84(\mathrm{~d}$, $J=7.2 \mathrm{~Hz}, 1 \mathrm{H}, \mathrm{Py}), 8.51(\mathrm{~s}, 1 \mathrm{H}, \mathrm{CH}), 9.21(\mathrm{~d}, J=6.9 \mathrm{~Hz}$, $1 \mathrm{H}, \mathrm{Py}), 12.91(\mathrm{~s}, 1 \mathrm{H}, \mathrm{NH}) ;{ }^{13} \mathrm{C}$ NMR $(150 \mathrm{MHz}$, DMSO$\left.\mathrm{d}_{6}\right) \delta 21.35,116.43,120.79,125.15,125.88,128.06,129.29$, $129.33,131.62,134.52,138.66,140.59,149.13,150.21$, 154.21; MS (ESI), m/z: 315(M+1) ${ }^{+}$. Elemental anal. For $\mathrm{C}_{15} \mathrm{H}_{12} \mathrm{ClN}_{5} \mathrm{O}$ (\%), calculated: $\mathrm{C}, 57.42 ; \mathrm{H}, 3.86 ; \mathrm{N}, 22.32$; found: C, 57.38; $\mathrm{H}, 4.01 ; \mathrm{N}, 22.11$.

8-Chloro-N'-(4-(trifluoromethyl)benzylidene)-[1,2,4] triazolo[4,3-a]pyridine-3-carbohydrazide (4p) white yellow crystal, yield $92 \%$, m.p. $>300{ }^{\circ} \mathrm{C}$; FT-IR $\left(\mathrm{KBr}, \mathrm{cm}^{-1}\right)$ : v 3321, 3153, 1673, 1620, 1545, 1523, 1487, 1449, 1331, $1297,1216,1153,1116,1069,1018,952,838,794,743 ;{ }^{1} \mathrm{H}$ NMR (DMSO-d $\left.{ }_{6}, 500 \mathrm{MHz}\right), \delta: 7.28(\mathrm{t}, J=7.2 \mathrm{~Hz}, 1 \mathrm{H}, \mathrm{Py})$, 7.82-7.85(m, $3 \mathrm{H}, \mathrm{Ar}$ and Py), 7.97(d, $J=8.0 \mathrm{~Hz}, 2 \mathrm{H}, \mathrm{Ar})$ $8.72(\mathrm{~s}, 1 \mathrm{H}, \mathrm{CH}), 9.21(\mathrm{~d}, J=6.9 \mathrm{~Hz}, 1 \mathrm{H}, \mathrm{Py}), 13.02(\mathrm{~s}, 1 \mathrm{H}$, $\mathrm{NH}) ;{ }^{13} \mathrm{C}$ NMR (150 MHz, DMSO-d 6 ) $\delta 116.55,120.82$, $125.89,126.21,126.31,128.36,129.46,138.53,140.51$, 148.32, 148.36, 149.20, 154.44; ${ }^{19} \mathrm{~F}$ NMR (564 MHz, DMSO- $\left._{6}\right) \delta-61.19 ;$ MS (ESI), m/z: 368(M+1) ${ }^{+}$. Elemental anal. For $\mathrm{C}_{15} \mathrm{H}_{9} \mathrm{ClF}_{3} \mathrm{~N}_{5} \mathrm{O}$ (\%), calculated: $\mathrm{C}$, 48.99; $\mathrm{H}$, 2.47; N, 19.05; found: C, 49.21; H, 2.72; N, 18.88 .

8-Chloro- $N^{\prime}$-(4-methylbenzylidene)-[1,2,4]triazolo[4,3a]pyridine-3-carbohydrazide (4q) white yellow crystal, yield $88 \%$, m.p. $>300{ }^{\circ} \mathrm{C}$; FT-IR $\left(\mathrm{KBr}, \mathrm{cm}^{-1}\right): v 3294$, 3143, 1694, 1671, 1605, 1541, 1509, 1488, 1459, 1357, $1233,1220,1152,1074,1043,953,910,842,813,743 ;{ }^{1} \mathrm{H}$ NMR (DMSO-d $\left.{ }_{6}, 500 \mathrm{MHz}\right), \delta: 2.36\left(\mathrm{~s}, 3 \mathrm{H}, \mathrm{CH}_{3}\right), 6.68(\mathrm{t}$, $J=7.5 \mathrm{~Hz}, 1 \mathrm{H}, \mathrm{Py}), 7.65(\mathrm{~d}, J=7.5 \mathrm{~Hz}, 2 \mathrm{H}, \mathrm{Ar}), 7.85(\mathrm{~d}$, $J=7.4 \mathrm{~Hz}, 2 \mathrm{H}, \mathrm{Ar}), 8.07(\mathrm{~d}, J=4.4 \mathrm{~Hz}, 1 \mathrm{H}, \mathrm{Py}), 8.51(\mathrm{~s}$, $1 \mathrm{H}, \mathrm{CH}), 9.21(\mathrm{~d}, J=6.9 \mathrm{~Hz}, 1 \mathrm{H}, \mathrm{Py}), 12.78(\mathrm{~s}, 1 \mathrm{H}, \mathrm{NH})$; ${ }^{13} \mathrm{C}$ NMR $\left(150 \mathrm{MHz}, \mathrm{DMSO}-\mathrm{d}_{6}\right) \delta 21.55,116.41,120.78$, $125.88,127.77,129.32,130.01,131.06,140.62,140.81$, 149.12, 150.17, 154.15; MS (ESI), m/z: $314(\mathrm{M}+1)^{+}$. Elemental anal. For $\mathrm{C}_{15} \mathrm{H}_{12} \mathrm{ClN}_{5} \mathrm{O}$ (\%), calculated: $\mathrm{C}, 57.42$; $\mathrm{H}, 3.86 ; \mathrm{N}, 22.32$; found: $\mathrm{C}, 57.65 ; \mathrm{H}, 3.03 ; \mathrm{N}, 22.55$.

\section{Antifungal activity}

Antifungal activities of compounds $\mathbf{4 a - 4 q}$ against Stemphylium lycopersici (Enjoji) Yamamoto, Fusarium oxysporum. sp. Cucumebrium and Botrytis cinerea were determined according to our previous work [31]. The potted plants cucumber and tomato were used. The determine concentration of control zhongshengmycin, thiophanate-methyl, cyprodinil and the title compounds is $100 \mu \mathrm{g} / \mathrm{mL}$. The three fungals Stemphylium lycopersici (Enjoji) Yamamoto, Fusarium oxysporum. sp. Cucumebrium and Botrytis cinerea were inoculated when the cucumber or tomato is at the stage of two seed leaves. The relative control efficacy of compounds comparing to the blank assay was calculated via the following equation:

$$
\text { Relative control efficacy }(\%)=(C K-P T) / C K \times 100 \%
$$

where $\mathrm{CK}$ is the average disease index during the blank assay and PT is the average disease index after treatment during testing. All experiments were replicated three times.

\section{Therotical calculations}

The theoretical calculation was carried out using DFT methods. The geometry optimization of compound $\mathbf{4 c}$ and $4 \mathbf{k}$ was carried out at the B3LYP/6-31G level. The energies of HOMO, LUMO and total energy, energy gap are calculated. All these are carried out using the Gaussian 03 package [32] on the dell computer. The LogP was calculated by Chemdraw 7.0.

\section{Conclusions}

In conclusion, a series of novel 1,2,4-triazolo[4,3-a]pyridine derivatives containing hydrazone moiety have been designed by bio-rational method based on the former lead compound by us. Many compounds were found to show good antifungal activity. The further comprehensive structure-active relationship was described by using theoretical calculation method. Among them, compound 4k possessed excellent antifungal activities against Stemphylium lycopersici (Enjoji) Yamamoto and Fusarium oxysporum. sp. Cucumebrium.

\section{Authors' contributions}

JXM, YXS, HKW, MYY and ZHS carried out experimental work, JXM prepared the manuscript, $\mathrm{XHL}, \mathrm{BJ}$ designed the material and supervised the project. All authors read and approved the final manuscript.

\section{Acknowledgements}

This work was supported financially by Zhejiang Provincial Natural Science Foundation of China (No. LY16C140007) and National Natural Science Foundation of China (No. 21205109) and we also thank Dr. Na-Bo Sun do some FTIR.

\section{Competing interests}

The authors declare that they have no competing interests.

Received: 17 February 2016 Accepted: 27 July 2016 Published online: 04 August 2016

References

1. Yan SL, Yang MY, Sun ZH, Min LJ, Tan CX, Weng JQ, Wu HK, Liu XH (2014) Synthesis and antifungal activity of 1,2,3-thiadiazole derivatives containing 1,3,4-thiadiazole moiety. Lett Drug Des Discov 11:940-943 
2. Liu XH, Weng JQ, Wang BL, Li YH, Tan CX, Li ZM (2014) Microwave-assisted synthesis of novel fluorinated 1,2,4-triazole derivatives, and study of their biological activity. Res Chem Intermed 40:2605-2612

3. Zhang $L$, Yang MY, Sun ZH, Tan CX, Weng JQ, Wu HK, Liu XH (2014) Synthesis and antifungal activity of 1,3,4-thiadiazole derivatives containing pyridine group. Lett Drug Des Discov 11:1107-1111

4. Cihan-Ustundag G, Simsek B, Ilhan E, Capan G (2014) Synthesis, characterization, antimycobacterial and anticancer evaluation of new 1,2,4-triazole derivatives. Lett Drug Des Discov 11:290-296

5. Muroga T, Sakaguchi T, Hashimoto T (2012) Synthesis and photoluminescence properties of heterocycle-containing poly(disubstituted acetylene) s. Polymer 53:4380-4387

6. Pandey D, Narvi SS, Mehrotra GK, Butcher RJ (2015) Hydrogen bonded 3D molecular self assembly constructed from [(Ni(nicotinamide)(2)(thiocyanate) $\left.(2)\left(\mathrm{H}_{2} \mathrm{O}\right)(2)\right]$ complex showing spin canted anti-ferromagnetic character. Chin J Struct Chem 34:777-785

7. Shapiro R, DiCosimo R, Hennessey SM, Stieglitz B, Campopiano O, Chiang GC (2001) Discovery and development of a commercial synthesis of azafenidin. Org Process Res Dev 5:593-598

8. Sun NB, Fu JQ, Weng JQ, Jin JZ, Tan CX, Liu XH (2013) Microwave assisted synthesis, antifungal activity and DFT theoretical study of some novel 1,2,4-triazole derivatives containing the 1,2,3-thiadiazole moiety. Molecules 18:12725-12739

9. Yang MY, Zhao W, Liu XH, Tan CX, Weng JQ (2015) Synthesis, crystal structure and antifungal activity of 4-(5-((2,4-dichlorobenzy)thio)-4-phenyl-4h-1,2,4-triazol-3-yl)pyridine. Chin J Struct Chem 34:203-207

10. Liu XH, Tan CX, Weng JQ (2011) Synthesis, dimeric crystal, and fungicidal activity of 1- (4-methylphenyl)-2-(5-((3,5-dimethyl-1 H-pyrazol-1-yl) methyl)-4-phenyl-4H-1,2,4-triazol-3-ylthio)ethanone. Phosphorus Sulfur Silicon Relat Elem 186:558-564

11. Liu XH, Xu XY, Tan CX, Weng JQ, Xin JH, Chen J (2015) Synthesis, crystal structure, herbicidal activities and 3D-QSAR study of some novel 1,2,4-triazolo[4,3-a]pyridine derivatives. Pest Manag Sci 71:292-301

12. Liu XH, Zhai ZW, Xu XY, Yang MY, Sun ZH, Weng JQ, Tan CX, Chen J (2015) Facile and efficient synthesis and herbicidal activity determination of novel 1,2,4-triazolo[4,3-a]pyridin-3(2H)-one derivatives via microwave irradiation. Bioorg Med Chem Lett 25:5524-5528

13. Yang MY, Zhai ZW, Sun ZH, Yu SJ, Liu XH, Weng JQ, Tan CX, Zhao WG (2015) A facile one-pot synthesis of novel 1,2,4-triazolo[4,3-a]pyridine derivatives containing the trifluoromethyl moiety using microwave irradiation. J Chem Res 39:521-523

14. Liu XH, Sun ZH, Yang MY, Tan CX, Weng JQ, Zhang YG, Ma Y (2014) Microwave assistant one pot synthesis, crystal structure, antifungal activities and 3D-QSAR of novel 1,2,4-triazolo[4,3-a] pyridines. Chem Biol Drug Des 84:342-347

15. Guan LP, Zhang RP, Sun $Y$, Chang Y, Sul X (2012) Synthesis and studies on the anticonvulsant activity of 5-alkoxy-[1,2,4]triazolo[4,3-a]pyridine derivatives. Arzneimittelforschung Drug Res 62:372-377

16. Sadana AK, Mirza Y, Aneja KR, Prakash O (2003) Hypervalent iodine mediated synthesis of 1-aryl/hetryl-1,2,4-triazolo[4,3-a] pyridines and 1-aryl/ hetryl 5-methyl-1,2,4-triazolo[4,3-a] quinolines as antibacterial agents. Eur J Med Chem 38:533-536

17. Li WQ, Zhang ZJ, Nan X, Liu YQ, Hu GF, Yu HT, Zhao XB, Wu D, Yan LT (2014) Design, synthesis and bioactivity evaluation of novel benzophenone hydrazone derivatives. Pest Manag Sci 70:667-673

18. Wang H, Ren SX, He ZY, Wang DL, Yan XN, Feng JT, Zhang X (2014) Synthesis, antifungal activities and qualitative structure activity relationship of carabrone hydrazone derivatives as potential antifungal agents. Int J Mol Sci 15:4257-4272

19. Kaplancikli ZA, Altintop MD, Ozdemir A, Turan-Zitouni G, Goger G, Demirci F (2014) Synthesis and in vitro evaluation of some hydrazone derivatives as potential antibacterial agents. Lett Drug Des Discov 11:355-362

20. Yang XD (2008) Synthesis and biological activity of hydrazone derivatives containing pyrazole. J Chem Res 9:489-491

21. Weng JQ, Wang L, Liu XH (2012) Synthesis, crystal structure and herbicidal activity of a 1, 2, 4-triazol-5(4h)-one derivative. J Chem Soc Pak 34:1248-1252
22. Liu XH, Tan CX, Weng JQ (2011) Phase transfer-catalyzed, one-pot synthesis of some novel $N$-pyrimidinyl- $N^{\prime}$-nicotinylthiourea derivatives. Phosphorus Sulfur Silicon Relat Elem 186:552-557

23. Liu XH, Zhao W, Shen ZH, Xing JH, Yuan J, Yang G, Xu TM, Peng WL (2016) Synthesis, nematocidal activity and docking study of novel chiral 1-(3-chloropyridin-2-yl)-3-(trifluoromethyl)-1 H-pyrazole-4- carboxamide derivatives. Bioorg Med Chem Lett 26:3626-3628

24. Liu XH, Pan L, Ma Y, Weng JQ, Tan CX, Li YH, Shi YX, Li BJ, Li ZM, Zhang YG (2011) Design, synthesis, biological activities, and 3D-QSAR of new $N$, $N^{\prime}$-diacylhydrazines containing 2-(2,4-dichlorophenoxy) propane moiety. Chem Biol Drug Des 78:689-694

25. Zhai ZW, Yang MY, Sun ZH, Liu XH, Weng JQ, Tan CX (2015) Facile and efficient synthesis of novel 1,2,3-thiadiazole derivatives using microwave irradiation. J Chem Res 39:340-342

26. Zhao W, Shen ZH, Xu TM, Peng WL, Liu XH (2016) Synthesis, nematocidal activity and docking study of novel chiral 1-(3-chloropyridin-2-yl)3-(difluoromethyl)-1H-pyrazole-4-carboxamide derivatives. J Heterocycl Chem. doi:10.1002/jhet.2753

27. Liu XH, Wang Q, Sun ZH, Wedge DE, Becnel JJ, Estep AS, Tan CX, Weng JQ (2016) Synthesis and insecticidal activity of novel pyrimidine derivatives containing urea pharmacophore against Aedes aegypti. Pest Manag Sci. doi:10.1002/ps.4370

28. Zhang LJ, Yang MY, Hu BZ, Sun ZH, Liu XH, Weng JQ, Tan CX (2015) Microwave-assisted synthesis of novel 8-chloro-[1,2,4]triazolo[4,3-a] pyridine derivatives. Turk J Chem 39:867-873

29. Shen ZH, Shi YX, Yang MY, Sun ZH, Weng JQ, Tan CX, Liu XH, Li BJ, Zhao WG (2016) Synthesis, crystal structure, dft studies and biological activity of a novel schiff base containing triazolo[4,3-a]pyridine moiety. Chin J Struct Chem 35:457-464

30. Zhai ZW, Shi YX, Yang MY, Sun ZH, Weng JQ, Tan CX, Liu XH, Li BJ, Zhang YG (2016) Synthesis, crystal structure, dft studies and antifungal activity of 5-(4-cyclopropyl-5-((3-fluorobenzyl)sulfonyl)-4H-1,2,4-triazol-3-yl)4-methyl-1,2,3-thiadiazole. Chin J Struct. Chem 35:25-33

31. Zhai ZW, Shi YX, Yang MY, Zhao W, Sun ZH, Weng JQ, Tan CX, Liu XH, Li BJ, Zhang YG (2016) Microwave assisted synthesis and antifungal activity of some novel thioethers containing 1,2,4-triazolo[4,3-a]pyridine moiety. Lett Drug Des Discov 13:521-525

32. Frisch M-J, Trucks G-W, Schlegel H-B, Scuseria G-E, Robb M-A, Cheeseman J-R, Montgomery J-A Jr, Vreven T, Kudin K-N, Burant J-C, Millam J-M, lyengar S-S, Tomasi J, Barone V, Mennucci B, Cossi M, Scalmani G, Rega N, Petersson G-A, Nakatsuji H, Hada M, Ehara M, Toyota K, Fukuda R, Hasegawa J, Ishida M, Nakajima T, Honda Y, Kitao O, Nakai H, Klene M, Li X, Knox J-E, Hratchian H-P, Cross J-B, Adamo C, Jaramillo J, Gomperts R, Stratmann R-E, Yazyev O, Austin A-J, Cammi R, Pomelli C, Ochterski J-W, Ayala P-Y, Morokuma K, Voth G-A, Salvador P, Dannenberg J-J, Zakrzewski V-G, Dapprich S, Daniels A-D, Strain M-C, Farkas O, Malick D-K, Rabuck A-D, Raghavachari K, Foresman J B, Ortiz J V, Cui Q, Baboul A G, Clifford S, Cioslowski J, Stefanov B-B, Liu G, Liashenko A, Piskorz P, Komaromi I, Martin R-L, Fox D-J, Keith T, Al-Laham M-A, Peng C-Y, Nanayakkara A, Challacombe M, Gill P-M-W, Johnson B, Chen W, Wong M-W, Gonzalez C, Pople J-A (2004) Gaussian 03, Revision C. 01. Gaussian Inc, Wallingford

\section{Submit your manuscript to a SpringerOpen ${ }^{\circ}$ journal and benefit from:}

- Convenient online submission

- Rigorous peer review

- Immediate publication on acceptance

- Open access: articles freely available online

- High visibility within the field

- Retaining the copyright to your article

Submit your next manuscript at springeropen.com 